\title{
Common Alzheimer's Disease Risk Variant within the $C L U$ Gene Affects White Matter Microstructure in Young Adults
}

\author{
Meredith N. Braskie, ${ }^{1 \star}$ Neda Jahanshad, ${ }^{1,2 \star}$ Jason L. Stein, ${ }^{1 \star}$ Marina Barysheva, ${ }^{1}$ Katie L. McMahon, ${ }^{4}$ \\ Greig I. de Zubicaray, ${ }^{5}$ Nicholas G. Martin, ${ }^{6}$ Margaret J. Wright, ${ }^{6}$ John M. Ringman, ${ }^{3}$ Arthur W. Toga, ${ }^{1}$ \\ and Paul M. Thompson ${ }^{1}$ \\ ${ }^{1}$ Laboratory of Neuro Imaging, Department of Neurology, ${ }^{2}$ Medical Imaging Informatics, Department of Radiology, and ${ }^{3}$ Mary S. Easton Center for \\ Alzheimer's Disease Research, Department of Neurology, University of California, Los Angeles, School of Medicine, Los Angeles, California 90095, ${ }^{4}$ Centre \\ of Advanced Imaging and ${ }^{5}$ School of Psychology, University of Queensland, Brisbane, Queensland 4072, Australia, and ${ }^{6}$ Queensland Institute of Medical \\ Research, Brisbane, Queensland 4029, Australia
}

There is a strong genetic risk for late-onset Alzheimer's disease (AD), but so far few gene variants have been identified that reliably contribute to that risk. A newly confirmed genetic risk allele $\mathrm{C}$ of the clusterin $(C L U)$ gene variant rs 11136000 is carried by $\sim 88 \%$ of Caucasians. The $\mathrm{C}$ allele confers a 1.16 greater odds of developing late-onset $\mathrm{AD}$ than the $\mathrm{T}$ allele. $\mathrm{AD}$ patients have reductions in regional white matter integrity. We evaluated whether the $C L U$ risk variant was similarly associated with lower white matter integrity in healthy young humans. Evidence of early brain differences would offer a target for intervention decades before symptom onset. We scanned 398 healthy young adults (mean age, $23.6 \pm 2.2$ years) with diffusion tensor imaging, a variation of magnetic resonance imaging sensitive to white matter integrity in the living brain. We assessed genetic associations using mixed-model regression at each point in the brain to map the profile of these associations with white matter integrity. Each C allele copy of the $C L U$ variant was associated with lower fractional anisotropy - a widely accepted measure of white matter integrity — in multiple brain regions, including several known to degenerate in $\mathrm{AD}$. These regions included the splenium of the corpus callosum, the fornix, cingulum, and superior and inferior longitudinal fasciculi in both brain hemispheres. Young healthy carriers of the $C L U$ gene risk variant showed a distinct profile of lower white matter integrity that may increase vulnerability to developing AD later in life.

\section{Introduction}

Two recent genome-wide association studies identified and replicated an association between risk for Alzheimer's disease (AD) and carrying the $\mathrm{C}$ allele of the single nucleotide polymorphism (SNP) rs11136000 in the clusterin (also known as apolipoprotein J) gene (CLU-C) (Harold et al., 2009; Lambert et al., 2009). $C L U-C$ confers a 1.16 greater odds of developing late-onset $\mathrm{AD}$ than the T allele (Bertram et al., 2007). Approximately 36\% of Caucasians carry two copies of the risk-conferring allele (Bertram et al., 2007), making this gene of great interest for public health.

Received Nov. 4, 2010; revised Jan. 25, 2011; accepted March 2, 2011.

Author contributions: K.L.M., G.I.d.Z., N.G.M., and M.J.W. collected the data; M.N.B., N.J., J.L.S., M.B., and P.M.T. analyzed the data; and M.N.B., N.J., J.L.S., and P.M.T. wrote the initial manuscript draft. All authors were involved in study design, discussed the results, and revised the manuscript.

This study was supported by the National Institute of Child Health and Human Development (Grant R01 HD050735) and the National Health and Medical Research Council (NHMRC) (Grant 486682), Australia. Genotyping was supported by NHMRC (Grant 389875). Additional support for algorithm development was provided by National Institutes of Health (NIH) R01 Grants EB008432, EB008281, and EB007813. M.N.B. was funded by the NIH (Grant T32 NS048004:05) and University of California, Los Angeles Easton Center for Alzheimer's Disease Research donor funds. N.J. was funded by the NIH/National Library of Medicine (Grant T15 LM07356). J.L.S. was also funded by the ARCS foundation and the National Institute of Mental Health (Grant 1F31MH087061).

*M.N.B., N.J., and J.L.S. contributed equally to the work.

Correspondence should be addressed to Dr. Paul M. Thompson, Laboratory of Neuro Imaging, Department of Neurology, UCLA School of Medicine, 635 Charles Young Drive South, Suite 225, Los Angeles, CA 90095-7334. E-mail: thompson@loni.ucla.edu.

DOI:10.1523/JNEUROSCI.5794-10.2011

Copyright $\odot 2011$ the authors $\quad 0270-6474 / 11 / 316764-07 \$ 15.00 / 0$
$\mathrm{AD}$ traditionally has been considered a disease marked by neuronal cell loss and widespread gray matter atrophy, but degeneration of myelin in white matter fiber pathways is increasingly considered a key disease component (Braak and Braak, 1996; Hua et al., 2008; Bartzokis, 2009). To evaluate how CLU-C affects fiber integrity, we scanned 398 healthy young adults (mean age, $23.6 \pm 2.2$ years) with diffusion tensor imaging (DTI). DTI is a variant of standard brain magnetic resonance imaging (MRI) that is sensitive to fiber integrity and white matter microstructure. The most widely accepted DTI measure, fractional anisotropy (FA), evaluates the extent to which water diffusion is directionally constrained. Higher FA generally reflects preferential diffusion along more intact, heavily myelinated axons. Demyelination, neurological disease symptoms, and slowed nerve conduction have been associated with lower FA in white matter (Nucifora et al., 2007), suggesting that it may reflect reduced white matter integrity.

White matter pathways deteriorate in $\mathrm{AD}$ due to primary effects of impaired myelination and secondary effects of neuronal loss (Brun and Englund, 1986). In several studies of AD and mild cognitive impairment, cognitive impairment has been associated with reduced FA in the corpus callosum, fornix, cingulum, superior longitudinal fasciculus (SLF), and inferior longitudinal fasciculus (ILF) (Liu et al., 2009; Stricker et al., 2009). Additionally, the apolipoprotein $\mathrm{E}$ allele $\varepsilon 4$ (APOE4) - a widely confirmed genetic risk factor for late-onset $\mathrm{AD}$-is associated with lower FA in 
the parahippocampal white matter, splenium, and frontooccipital fasciculus of healthy older subjects (Nierenberg et al., 2005; Persson et al., 2006; Smith et al., 2010).

$C L U$ is a plausible candidate for modulating white matter integrity, as both $C L U$ and $A P O E$ encode apolipoproteins implicated in $\mathrm{AD}$ risk. Additionally, clusterin (the gene product) is implicated in lipid transport and membrane recycling (Dati et al., 2007), and in remyelination of nerve fibers in rats (Dati et al., 2007). We hypothesized that young CLU-C carriers would show reduced white matter integrity, quantifiable as lower FA in brain regions implicated in AD. Such differences could help to explain why the gene is associated with heightened risk for AD.

It is vital to discover how $\mathrm{AD}$ risk variants impact the living brain to better understand disease development and to design interventions for those at risk. Targeting subpopulations most likely to show decline also boosts power for prevention and treatment trials (Kohannim et al., 2010).

\section{Materials and Methods}

Subjects and genotype information. DTI scans and genotypes were obtained for 468 right-handed Caucasians recruited as part of a project examining brain structure and white matter integrity in healthy, young adult Australian twins. We excluded 70 subjects for the following reasons: 8 were ancestry outliers; 58 were technically inadequate scans; 3 had ventricle size inconsistent with good health in a young person; and 1 lacked a genotype at rs 11136000 . Of the remaining 398 subjects (mean age, $23.6 \pm 2.2$ years; age range, $20-29$ years), 92 were monozygotic (MZ) twins, 147 were dizygotic (DZ) twins or triplets, 41 were singleton siblings, and 118 were unrelated individuals. Genomic DNA samples were analyzed on the Human610-Quad BeadChip (Illumina) according to the manufacturer's protocols (Infinium HD Assay; Super Protocol Guide, revision A, May 2008). The CLU rs 11136000 polymorphism was $\mathrm{T} / \mathrm{T}$ in 68 subjects (17.1\%), $\mathrm{C} / \mathrm{T}$ in 220 subjects $(55.3 \%)$, and $\mathrm{C} / \mathrm{C}$ in 110 subjects $(27.6 \%)$. The frequency for the minor (T) allele (MAF) in unrelated subjects in our sample was 0.40 , similar to previous reports in healthy populations of European origin (Harold et al., 2009; Lambert et al., 2009). The genotype distribution in our sample followed HardyWeinberg equilibrium using a standard threshold of $p>0.001\left(\chi_{(1)}^{2}=\right.$ $4.70, p=0.030)$. When we calculated the evidence for Hardy-Weinberg equilibrium, the expected proportions of $\mathrm{C} / \mathrm{C}$ and $\mathrm{T} / \mathrm{T}$ genotypes compared with observed values differed by small numbers that can be attributed to the effects of random sampling. These differences are unlikely to indicate any ascertainment bias as these subjects were selected only because they are twins or singleton siblings of twins. They were originally recruited for a melanoma study, and the cohort is composed of young adults, meaning that there would not be any disease-related attrition that could adversely impact the allele frequencies for an $\mathrm{AD}$ risk allele. We therefore have no good reason to believe that those in the study have a level of $\mathrm{AD}$ risk that is different from that of the general Australian population from which our sample was drawn.

Verbal, performance, and full-scale intelligence quotient (IQ) standardized scores were derived from subtest scores of the Multidimensional Aptitude Battery (MAB) (Jackson, 1984), which were available for all but 20 subjects. The MAB is similar to the Wechsler Adult Intelligence Scale (Wechsler, 1981) as it is composed of tests of verbal and performance intelligence. Verbal IQ subtests included assessments of information, arithmetic, and vocabulary. Performance IQ subtests included spatial and object assembly. All subjects provided written, informed consent. The study conformed to the National Statement on Ethical Conduct in Human Research (2007) issued by the National Health and Medical Research Council of Australia and was approved by the Queensland Institute of Medical Research Human Research Ethics Committee.

Image acquisition. T1-weighted images of the brain were acquired with an inversion recovery rapid gradient echo sequence on a 4 Tesla MRI scanner (Medspec, Bruker) [acquisition parameters: inversion time, 700 $\mathrm{ms}$; repetition time (TR), $1500 \mathrm{~ms}$; echo time (TE), $3.35 \mathrm{~ms}$; flip angle = $8^{\circ}$; slice thickness $\left.=0.9 \mathrm{~mm} ; 256 \times 256 \times 256\right]$. Diffusion-weighted images were acquired using single-shot echo planar imaging (EPI) with a twice-refocused spin echo sequence to reduce eddy-current-induced distortions (TR, $6090 \mathrm{~ms}$; TE, $91.7 \mathrm{~ms}$; field of view, $23 \mathrm{~cm} ; 128 \times 128$ ). Each three-dimensional (3D) volume consisted of 55 axial slices $(2.0 \mathrm{~mm} / 0$ $\mathrm{mm}$ gap; $1.79 \times 1.79 \mathrm{~mm}$ in-plane resolution). We acquired 105 images per subject: 11 with no diffusion sensitization (i.e., T2-weighted $b_{0}$ images) and 94 diffusion-weighted (DW) images $\left(b=1149 \mathrm{~s} / \mathrm{mm}^{2}\right)$ with gradient directions evenly distributed on the hemisphere.

DTI preprocessing. We automatically removed nonbrain regions from the T1-weighted MR and DW $b_{0}$ images using FSL BET (http://fsl.fmrib. ox.ac.uk/fsl/), then manually refined the brain extraction. All T1weighted images were linearly aligned to a common space (with a global transform that had 9 degrees of freedom). The raw DW images were corrected for eddy current distortions using the FSL "eddy correct" method (http://fsl.fmrib.ox.ac.uk/fsl/). Individual $b_{0}$ images were averaged, linearly aligned, and resampled to their corresponding $\mathrm{T} 1$ images. The average $b_{0}$ maps were then elastically registered to the individual common space T1-weighted scans using a mutual information cost function (Leow et al., 2005) to control for EPI-induced susceptibility artifacts.

Computing fractional anisotropy. We compared FA values at each voxel across $C L U$ genotypes. Diffusion tensors were computed at each voxel using FSL software (http://fsl.fmrib.ox.ac.uk/fsl/). From the tensor eigenvalues $\left(\lambda_{1}, \lambda_{2}, \lambda_{3}\right)$, FA was calculated according to the following formula:

$$
\begin{gathered}
F A=\sqrt{\frac{3}{2}} \frac{\sqrt{\left(\lambda_{1}-\bar{\lambda}\right)^{2}+\left(\lambda_{2}-\bar{\lambda}\right)^{2}+\left(\lambda_{3}-\bar{\lambda}\right)^{2}}}{\sqrt{\lambda_{1}^{2}+\lambda_{2}^{2}+\lambda_{3}^{2}}} \\
\bar{\lambda}=\frac{\lambda_{1}+\lambda_{2}+\lambda_{3}}{3} .
\end{gathered}
$$$$
\in[0,1]
$$

We also analyzed radial diffusivity $\left(\mathrm{D}_{\mathrm{rad}}\right)$ (the average of $\lambda_{2}$ and $\lambda_{3}$ ) and axial diffusivity $\left(\mathrm{D}_{\mathrm{ax}}\right)\left(\lambda_{1}\right)$ to clarify the extent to which each might be contributing to the changes in FA.

Template creation and registration. We used nonlinear fluid registration (Lepore et al., 2008) to create a mean deformation target (MDT) from the FA images (calculated after $b_{0}$ susceptibility correction) (Jahanshad et al., 2010). Included in the MDT were 32 randomly selected unrelated subjects (16 female/16 male). The N 3D vector fields that fluidly registered a specific individual to all other $N$ subjects were averaged and applied to that subject, preserving the image intensities and anatomical features of the template subject.

Susceptibility-corrected FA maps were registered to the final population-averaged FA-based MDT using a 3D elastic warping technique with a mutual information cost function (Leow et al., 2005). To better align white matter regions of interest, the MDT and all wholebrain registered FA maps were thresholded at 0.25 (excluding contributions from non-white matter). Thresholded FA maps were then reregistered to the thresholded MDT and smoothed with a Gaussian kernel (9 $\mathrm{mm}$ full width at half-maximum). In this way, the outlines of the major white matter structures are stable and have been normalized to a very fine degree of matching across subjects, greatly reducing the neuroanatomical variations in these structures across subjects. To ensure that our results were not due to morphometric differences that were not fully corrected by the fluid registration, we additionally performed tissue-specific, smoothing-compensated voxel-based analysis (T-SPOON) (Lee et al., 2009), which reduces that confound.

Statistical analyses. We performed mixed-model regression at each voxel to model family relatedness (Kang et al., 2008). A symmetric $N \times N$ kinship matrix was constructed to describe the relationship of every subject to all others. A kinship matrix coefficient of 1 denoted the relationship of the subjects to themselves or their MZ twin: 0.5 indicated DZ twins and siblings within the same family, and 0 denoted unrelated subjects. Ancestry outliers were removed, so no additional modeling was used in the kinship matrix to adjust for population genetic structure between families. We then used a linear mixed-effects model to estimate the association of each copy of CLU-C in the SNP rs 11136000 to FA, $\mathrm{D}_{\mathrm{ax}}$, 
and $D_{\text {rad }}$ measures at each voxel, controlling for familial relatedness through the kinship matrix, age, and sex.

Our analysis used an additive model that assessed the effect of each risk allele rather than evaluating the effects of $C L U$-C carriers or noncarriers (i.e., we counted the number of adverse alleles and used that number in a regression across the full sample). The $C L U$ association with $\mathrm{AD}$ initially was discovered using a test for differences in allele frequencies between diagnostic groups. Here we use a quantitative phenotype, so that test cannot be used. However, the additive model we used (vs a recessive or dominant model) is the most powerful for finding genetic effects in a quantitative phenotype. To further investigate the nature of this relationship, we used post hoc exploratory tests to evaluate how CLU genotype related to FA using models other than the additive model to compare genotype groups, while controlling for age and sex. Specifically, we evaluated $\mathrm{C} / \mathrm{C}$ versus $\mathrm{T}, \mathrm{T} / \mathrm{T}$ versus $\mathrm{C}, \mathrm{C} / \mathrm{T}$ versus $\mathrm{T} / \mathrm{T}$, and $\mathrm{C} / \mathrm{T}$ versus $\mathrm{C} / \mathrm{C}$ carrier. This must be considered exploratory testing because we chose the additive model as our primary hypothesis since it is the most powerful model to use if the effects are additive.

To ensure that only white matter was being considered and to reduce the effects of partial voluming, only voxels in which $\mathrm{FA}>0.3$ in the MDT were considered in the statistical analyses. The $p$ value reported is based on the fixed-effect regression coefficient ( $\beta$ parameter) of the additive genetic effect (see Fig. 1). This analysis was performed using Efficient Mixed-Model Association (Kang et al., 2008) (EMMA; http://mouse.cs.ucla.edu/emma/) within the $\mathrm{R}$ statistical package (version 2.9.2; http://www.r-project.org/). We used the widely used false discovery rate (FDR) method to control for voxelwise multiple comparisons (Benjamini and Hochberg, 1995). Single-factor ANOVAs were used to evaluate whether genotype groups were different in age, sex, or IQ. We also controlled for age and sex in the full sample, and age, sex, and full IQ in a subsample of 378 subjects for whom IQ values were available.

\section{Results}

As shown in Table 1, genotype groups ( T/T, C/T, and C/C) were not significantly different in age $\left(F_{(2,395)}=1.30, p=0.27\right)$, verbal IQ $\left(F_{(2,375)}=0.31, p=0.73\right)$, performance IQ $\left(F_{(2,375)}=0.82, p=0.44\right)$, or full-scale IQ $\left(F_{(2,375)}=0.65, p=0.53\right)$, as tested using ANOVAs. Sex was not significantly different between genotype groups, as determined using a $\chi^{2}$ test $\left(\chi_{(2)}^{2}=0.84, p=0.66\right)$.

White matter integrity was quantified using the widely accepted index FA. In statistical maps based on 398 young adults, each $C L U-\mathrm{C}$ allele (adjusted for age and sex) was associated with lower FA in frontal, temporal, parietal, occipital, and subcortical white matter (multiple comparisons corrected: critical $p=0.023$, for an FDR controlled at 5\%; minimum $p=5.9 \times 10^{-7} ; 46.1 \%$ of evaluated voxels survived the FDR threshold). Brain regions with lower FA included corticocortical pathways previously demonstrated to have lower FA in $\mathrm{AD}$ patients and
Table 1. Subject characteristics by CLU rs11136000 genotype in the studied population

\begin{tabular}{llll}
\hline & \multicolumn{3}{l}{ Genotypes } \\
\cline { 2 - 4 } & $\mathrm{T} / \mathrm{T}$ & $\mathrm{C} / \mathrm{T}$ & $\mathrm{C} / \mathrm{C}$ \\
\hline Subjects & 68 & 220 & 110 \\
Sex & $24 \mathrm{M}, 44 \mathrm{~F}$ & $77 \mathrm{M}, 143 \mathrm{~F}$ & $44 \mathrm{M}, 66 \mathrm{~F}$ \\
Age & $23.6 \pm 2.3$ & $23.5 \pm 2.2$ & $23.9 \pm 2.3$ \\
No. of IQ test scores & 64 & 208 & 106 \\
PIQ & $112.4 \pm 17.0$ & $115.2 \pm 15.8$ & $114.0 \pm 14.4$ \\
VIQ & $111.3 \pm 10.8$ & $112.2 \pm 11.3$ & $112.7 \pm 10.0$ \\
FIQ & $112.8 \pm 12.9$ & $114.8 \pm 13.1$ & $114.5 \pm 11.1$ \\
\hline
\end{tabular}

Values are given as the mean \pm SD. Sex, age, and IQ scores are not significantly different between genotype group $(p>0.05)$ using ANOVAs to evaluate group differences in age and IQ, and a $\chi^{2}$ test to evaluate differences in sex. M, Male; F, female; PIQ, performance IQ; VIQ, verbal IQ; FIQ, full-scale IQ.
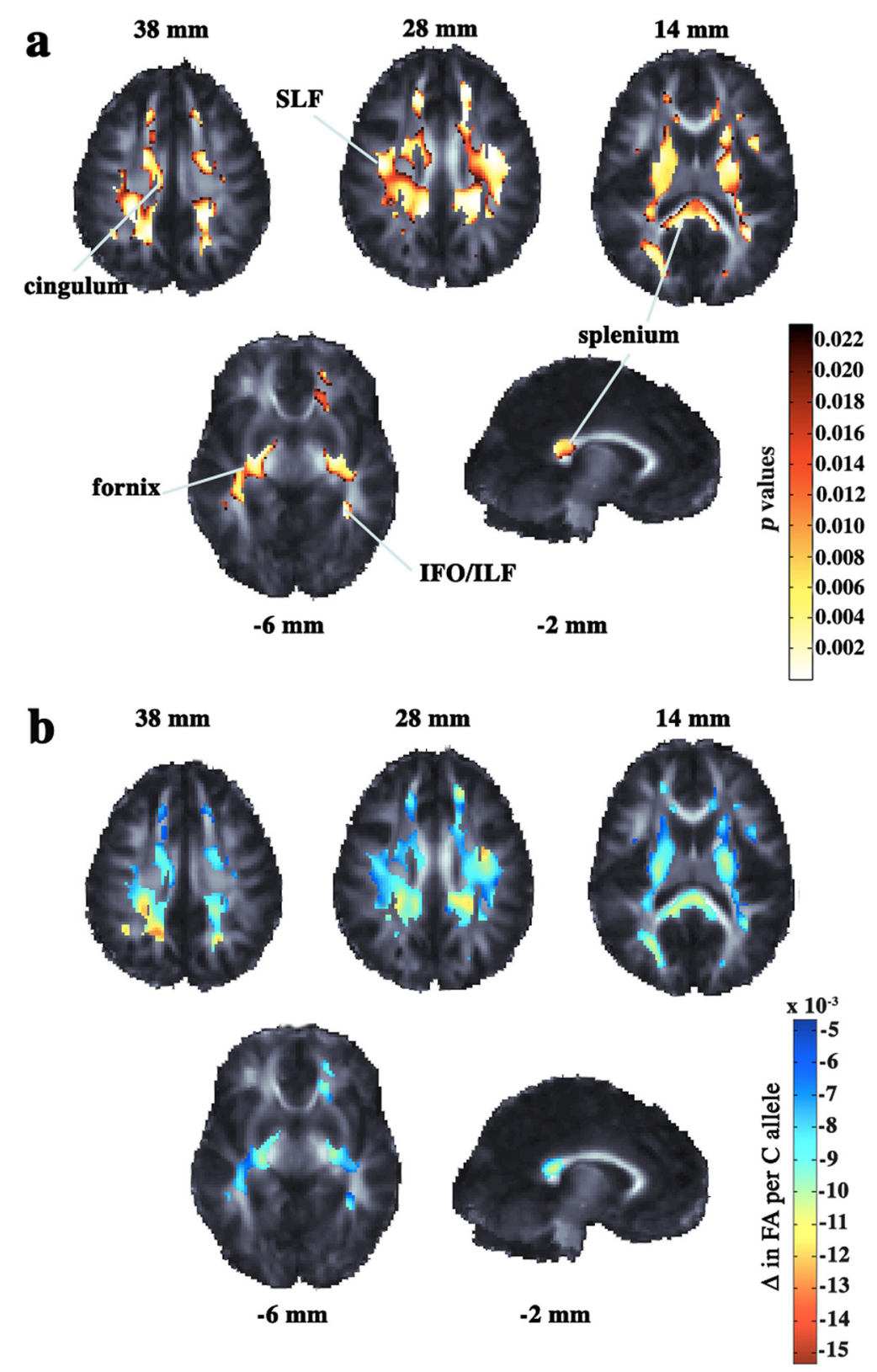

Figure 1. FA association with CLU-C displayed on a study-specific FA template. $\boldsymbol{a}$, Highlighted areas are the $p$ values indicating voxels in which CLU-C is associated with lower FA after adjusting for age and sex (FDR critical $p$ value $=0.023$ ). In $\boldsymbol{b}$, we show regression coefficients at significant voxels that encompass many regions that degenerate in $A D$. The left brain hemisphere is displayed on the right. Coordinates listed are for the $Z$ (for axial slices) and $X$ (for the sagittal slice) directions in ICBM space. SLF and ILF denote the superior and inferior longitudinal fasciculi. IFO is the inferior fronto-occipital fasciculus. 

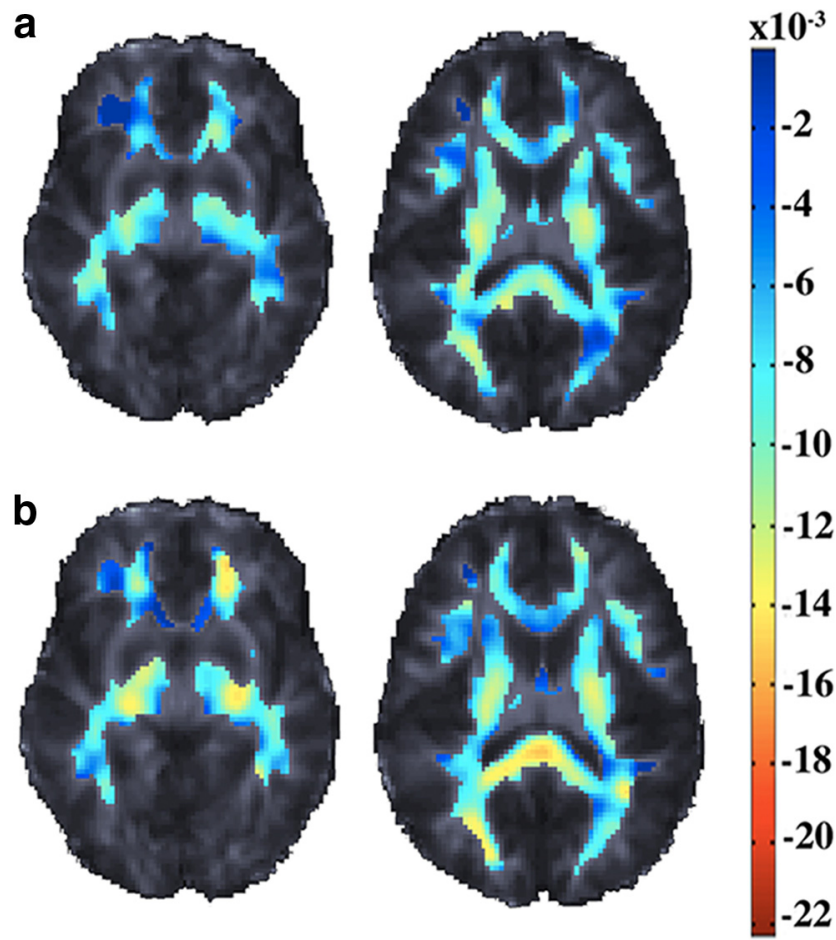

$-14$

$-16$

18

Figure 2. Statistical models. Highlighting represents un-normalized regression coefficients or beta values in all white matter voxels tested. It demonstrates the CLU-C association with lower FA (after adjusting for age and sex). $\boldsymbol{a}, \boldsymbol{b}$, Shown here as post hoc explorations are the $\mathrm{C} / \mathrm{C}$ versus $\mathrm{T}$ carrier model $(\boldsymbol{a})$ and the $\mathrm{T} / \mathrm{T}$ versus $\mathrm{C}$ carrier model $(\boldsymbol{b})$. Units are in FA unit difference between groups for these models, while they are in FA units per allele for the additive model (Fig. 1). The per-voxel effect is similar in location using all models, but only the initially hypothesized and most powerful additive model passes FDR correction, as shown in Figure 1 (FDR critical $p$ value $=0.023$ ). The effect appears to depend on the allele dose rather than the presence or absence of a given allele. The left brain hemisphere is displayed on the right.

APOE4 carriers. Strongest effects ( $\geq 0.01$ units FA reduction per risk allele) were found in the splenium, bilateral posterior and anterior corona radiata, bilateral perithalamic tracts, and posterior thalamic radiation, and complex association fibers [left SLF, and ILF/inferior fronto-occipital fasciculus (IFO)]. We also found significant associations bilaterally in peristriatal and periventricular white matter, other callosal fibers (corpus callosum body and genu, and forceps major and minor), limbic tracts (fornix, stria terminalis, and posterior cingulum, including in the parahippocampal white matter), complex association fibers (SLF, ILF/IFO), and tracts of the cerebral peduncles (Fig. 1). Our results remained significant when adjusted for age, sex, and fullscale IQ in the 378 subjects for whom IQ values were available (multiple comparisons corrected: critical $p=0.028$, for an FDR controlled at $5 \%$; minimum $p=1.9 \times 10^{-7} ; 56.3 \%$ of evaluated voxels survived the FDR threshold).

FA was significantly correlated with $C L U$ genotype after FDR correction using the additive model. The additive model outperformed all the other models for comparing genotype categories, and therefore best explains the underlying effect in our sample. Using an uncorrected $p<0.05$ threshold for illustrative purposes only, we report the following when all subjects were considered: those with a T/T genotype showed regionally greater FA than $\mathrm{C}$ carriers in $36 \%$ of voxels considered at this liberal statistical threshold (minimum $p=8.9 \times 10^{-6}$ ). Those with a C/C genotype showed lower FA than T carriers in $40 \%$ of voxels considered at this liberal statistical threshold (minimum $p=7.0 \times 10^{-5}$ ) (Fig. 2). When 220 subjects with a $\mathrm{C} / \mathrm{T}$ genotype were compared with either 110 subjects with a C/C genotype or 68 with a T/T genotype, $14 \%\left(\right.$ minimum $p=3.6 \times 10^{-4}$ ) and $10 \%$ (minimum $p=3.6 \times 10^{-5}$ ) of considered voxels, respectively, were significant at an uncorrected $p<0.05$ level. Overall, this evidence favors an additive (allele dose-dependent) effect on the white matter.

FA is influenced by both $\mathrm{D}_{\mathrm{ax}}$ (a measure of diffusion along the axonal fibers) and $\mathrm{D}_{\text {rad }}$ (a measure of diffusion perpendicular to the axonal fibers). Reduced white matter integrity (as measured by FA) can sometimes be further traced back to greater $\mathrm{D}_{\text {rad }}$ or lower $\mathrm{D}_{\mathrm{ax}}$. To further investigate the reduced FA in CLU-C carriers, we examined the correlation of CLU genotype with $\mathrm{D}_{\text {rad }}$ and $\mathrm{D}_{\mathrm{ax}}$ on a voxelwise basis, while adjusting for age and sex. Each $C L U$-C allele was associated with increased $\mathrm{D}_{\text {rad }}$ (multiple comparisons corrected: critical $p=0.022$, for an FDR controlled at $5 \%$; minimum $p=0.0001 ; 44.3 \%$ of evaluated voxels survived the FDR threshold) after adjusting for age and sex (Fig. 3). $\mathrm{D}_{\mathrm{ax}}$ was not significantly associated with $C L U$ genotype.

DTI studies have reported lower FA in healthy older APOE4+ subjects in the parahippocampal white matter, splenium, and fronto-occipital fasciculus (Nierenberg et al., 2005; Persson et al., 2006; Smith et al., 2010). To determine whether our results were influenced by APOE genotypes, we evaluated the effects on DTI FA of a proxy or tag SNP for the APOE4 polymorphism rs2075650 within the TOMM40 gene, because the APOE4 polymorphism was not directly genotyped on the chip. The TOMM40 variant is in moderate linkage disequilibrium with APOE4 $\left(r^{2}=\right.$ 0.48) (Harold et al., 2009). Our CLU results remained significant after adjusting for age, sex, and TOMM40 genotype (corrected for multiple comparisons; critical $p=0.026$, for an FDR controlled at $5 \%$; minimum $p=6.1 \times 10^{-7} ; 51.1 \%$ of evaluated voxels survived the FDR threshold). These results confirm that the $C L U$-C effect on FA was not attributable to TOMM40 genotypes, and hence APOE4-associated changes.

Finally, to reduce the likelihood that coregistration errors contributed to our results, we performed an analysis known as T-SPOON (Lee et al., 2009) on our data using individual FA masks thresholded at FA $>0.25$. We then reran the primary statistical analysis, adjusting for age and sex, and using the same thresholding and spatial smoothing as in the primary analyses. The T-SPOON analysis adjusts for effects at the interfaces of white matter and other tissues. Our results remained significant (corrected for multiple comparisons; critical $p=0.019$, for an FDR controlled at 5\%; minimum $p=1.2 \times 10^{-5} ; 37.8 \%$ of evaluated voxels survived the FDR threshold) in all the same brain regions as our primary analysis. This is in line with expectation, as most of the differences were found far away from any gray matter and reflect differences in the characteristics of white matter in many regions without partial volume of multiple tissue types.

\section{Discussion}

We found widespread lower FA in the white matter of healthy young adults who carry a recently identified risk gene for lateonset Alzheimer's disease. Effects occurred in multiple regions, including several known to degenerate in AD. Such regions included the corpus callosum, fornix, cingulum, SLF, and ILF (Liu et al., 2009; Stricker et al., 2009). This suggests that the CLU-C related variability found here might create a local vulnerability important for disease onset. These effects are remarkable as they already exist early in life and are associated with a risk gene that is very prevalent ( $\sim 36 \%$ of Caucasians carry two copies of the riskconferring genetic variant $C L U-\mathrm{C}$ ).

Higher FA does not always imply better neuronal function, and there are neurogenetic syndromes where higher FA in some 
brain regions is associated with abnormal function (Hoeft et al., 2007). Accepting these as counterexamples, lower FA is generally a sign of poorer fiber coherence, myelination, and poorer function, as noted in a recent review of DTI studies across many domains of neuropsychiatry (Thomason and Thompson, 2011).

Lower FA may indicate reduced myelin integrity or axonal damage. We found a significant increase in $D_{\text {rad }}$ widely throughout the white matter without associated significant decreases in $\mathrm{D}_{\mathrm{ax}}$. These results suggest that reduced myelin integrity, rather than axonal degeneration, may be responsible for the lower FA we found in many of these regions ( $\mathrm{Di}$ Paola et al., 2010). Increased regional $D_{\text {rad }}$ has previously been demonstrated in $\mathrm{AD}$ patients versus controls (Choi et al., 2005; Stricker et al., 2009; Zhang et al., 2009; Di Paola et al., 2010; Salat et al., 2010) and in healthy $A P O E 4+$ subjects versus those who do not carry the APOE4 allele (Nierenberg et al., 2005). In AD patients, increased $\mathrm{D}_{\text {rad }}$ has been attributed to degeneration of the myelin sheath (Di Paola et al., 2010), but our demonstration of in-

creased $\mathrm{D}_{\text {rad }}$ in healthy young $C L U-\mathrm{C}$ carriers raises the question of whether the increased $\mathrm{D}_{\text {rad }}$ seen in $\mathrm{AD}$ patients in past studies may also stem in part from inadequate myelination that occurs developmentally as a result of genes that increase AD risk. This is not to say that reduced myelin integrity does not play a role in $\mathrm{AD}$, but rather that both developmental differences and age- or disease-related degeneration may contribute to that reduced integrity.

Genetic risk for reduced FA may increase the risk for later cognitive impairment through developmental insufficiency. A lesser degree of myelination in CLU-C carriers may arise during development, which may not translate into poorer cognition in youth as the brain can compensate via redundant functionality. However, when exacerbated by other factors, such as age-related neuronal atrophy, and plaque and tangle burden in $\mathrm{AD}$, reduced myelin integrity could facilitate cognitive impairment. As our study examined how a common $\mathrm{AD}$ risk gene affects young adults who have no observable cognitive deficits, it is unlikely that we are seeing the earliest possible signs of $\mathrm{AD}$-associated brain changes. More likely, the reduced fiber integrity represents an early developmental vulnerability that may reduce brain resilience to later $\mathrm{AD}$ pathology; in other words, its mechanism of action may not be part of the classic AD pathways that lead to abnormal amyloid plaque and neurofibrillary tangle accumulation in the brain.

Lower FA in late-onset Alzheimer's disease may be promoted by suboptimal amyloid processing in the brain. Amyloid plaques and neurofibrillary tangles - the primary pathological hallmarks of $\mathrm{AD}$ - accumulate in the brain decades before symptoms appear. Neurofibrillary tangles are detectable in $\sim 20 \%$ of subjects aged 26-35 years (Braak and Braak, 1997), and greater amyloid deposition in healthy elderly subjects is correlated with greater neuropsychological decline over the preceding decade (Resnick et al., 2010). Clusterin is found in amyloid plaques (Calero et al., 1999 ) and transports soluble $\beta$-amyloid (A $\beta$ ) across the blood- brain barrier into brain parenchyma (Zlokovic et al., 1996). A $\beta$ then may damage the oligodendrocytes, which generate myelin, as reported in vitro (Roth et al., 2005). However, in our young healthy sample, lower FA may reflect variability in lipid processing as the lipid-rich myelin sheath develops; it is unlikely to be evidence of a disease mechanism or a biomarker of AD. Myelin abnormalities and axonal swelling may contribute to synaptic loss and precede amyloid deposition in AD (Bartzokis, 2009). If so, the $C L U$ risk variant could increase AD risk in two ways: an early acquired vulnerability paired with suboptimal amyloid processing in later life.

Although the additive model we used assesses evidence for an aggregate risk of carrying increasing numbers of alleles, the associated cellular processes that result in lower FA are not necessarily the primary pathways by which the SNP confers AD risk. Rather, our findings suggest one way in which vulnerability to AD may be increased.

Thus far, only one neuroimaging study has examined brain differences in CLU-C carriers (Biffi et al., 2010). That study found that the $C L U$ genotypes were not associated with MRI measures. However, in that study structural MRI was used, which is less sensitive than DTI to altered fiber microstructure and myelination. The authors of that study noted that the effects on brain structure of different gene variants that increase $\mathrm{AD}$ risk may be specific to particular and disparate aspects of brain structure. This segregation of gene effect on neuroimaging traits can offer important insights into the mechanisms through which the polymorphisms impact AD risk (Biffi et al., 2010).

While 398 subjects would be a small sample on which to identify new genetic risk factors for AD using genome-wide association scanning, it is in fact the largest DTI study to date to examine the effects of Alzheimer's disease genetic risk factors on DTI FA. Prior studies found effects of APOE4 on FA in sample sizes that ranged from 29 to 69 (Nierenberg et al., 2005; Persson et al., 2006; Honea et al., 2009; Smith et al., 2010). The effects of familial AD 
genes were detected in only 20 adults (Ringman et al., 2007). Admittedly, the odds ratios for those risk factors are greater than for $C L U$. However, our statistical power was boosted not only by our much larger sample size of 398 subjects, but also by our scanning at a stronger field strength ( $4 \mathrm{~T}$, as opposed to 1.5 or $3 \mathrm{~T}$ in the previous studies) and with more diffusion-weighted gradients ( 94 in our study vs 6 or 12 in previous studies). It is therefore not surprising that we had the power to detect the existing effect.

Although the $C L U$ risk variant was a candidate gene, whose effects we set out here to assess, one may also consider the value of making a correction, across studies, for examining multiple $\mathrm{AD}$ risk genes. Because of the very strict voxelwise corrections for multiple comparisons required in imaging genetics when using FDR, the rates of false positives (even when examining several hundred SNPs that are not expected to have a significant relationship with the data) remains well below $0.05(0.2-4.1 \%$ for 720 SNPs), as determined empirically (Meyer-Lindenberg et al., 2008). The fact that the $C L U$ rs 11136000 is a candidate variant chosen a priori based on its relationship to lipid transport and Alzheimer's disease makes it unlikely that our strong results were due to false positives.

We do not yet have available to us a comparably large dataset in which to independently replicate our results. This remains a limitation of our study as genetic studies typically employ very large samples, and, where possible, they replicate effects to avoid the risk of false discoveries. Continued data collection and collaborative efforts that allow for larger sample sizes will remedy this in the future. However, our results remain valuable as a focus for ongoing efforts by our group and others.

Quantitative mapping of structural brain differences in those at genetic risk for $\mathrm{AD}$ is crucial for evaluating treatment and prevention strategies. Once identified, brain differences can be monitored to determine how lifestyle choices influence brain health and disease risk. Many lifestyle factors that heighten the risk for dementia - such as exercise and body mass index- have effects on brain structure and the level of brain atrophy (Ho et al., 2010b,c; Raji et al., 2010). Additionally, regular exercise and a healthful diet may reduce the risk of cognitive decline, particularly in those genetically at risk for AD (Rovio et al., 2005; Scarmeas et al., 2009) or those carrying common risk alleles generally associated with brain structure deficits in healthy adults (Ho et al., 2010a). Targeting adults at greatest risk for cognitive deterioration can also improve the power of clinical trials (Kohannim et al., 2010). Future DTI studies of CLU-C in those imaged with amyloid- or tangle-sensitive positron emission tomography probes will also help to relate lower white matter integrity to $\mathrm{AD}$ pathology as it emerges.

\section{References}

Bartzokis G (2009) Alzheimer's disease as homeostatic responses to agerelated myelin breakdown. Neurobiol Aging. Advance online publication. Retrieved April 11, 2011. doi:10.1016/j.neurobiolaging.2009.08.007.

Benjamini Y, Hochberg Y (1995) Controlling the false discovery rate- a practical and powerful approach to multiple testing. J R Stat Soc Series B Stat Methodol 57:289-300.

Bertram L, McQueen MB, Mullin K, Blacker D, Tanzi RE (2007) Systematic meta-analyses of Alzheimer disease genetic association studies: the AlzGene database. Nat Genet 39:17-23.

Biffi A, Anderson CD, Desikan RS, Sabuncu M, Cortellini L, Schmansky N, Salat D, Rosand J (2010) Genetic variation and neuroimaging measures in Alzheimer disease. Arch Neurol 67:677-685.

Braak H, Braak E (1996) Development of Alzheimer-related neurofibrillary changes in the neocortex inversely recapitulates cortical myelogenesis. Acta Neuropathol 92:197-201.
Braak H, Braak E (1997) Frequency of stages of Alzheimer-related lesions in different age categories. Neurobiol Aging 18:351-357.

Brun A, Englund E (1986) A white matter disorder in dementia of the Alzheimer type: a pathoanatomical study. Ann Neurol 19:253-262.

Calero M, Tokuda T, Rostagno A, Kumar A, Zlokovic B, Frangione B, Ghiso J (1999) Functional and structural properties of lipid-associated apolipoprotein J (clusterin). Biochem J 344:375-383.

Choi SJ, Lim KO, Monteiro I, Reisberg B (2005) Diffusion tensor imaging of frontal white matter microstructure in early Alzheimer's disease: a preliminary study. J Geriatr Psychiatry Neurol 18:12-19.

Dati G, Quattrini A, Bernasconi L, Malaguti MC, Antonsson B, Nicoletti F, Alliod C, Di Marco R, Sagot Y, Vitte PA, Hiver A, Greco B, Roach A, Zaratin PF (2007) Beneficial effects of r-h-CLU on disease severity in different animal models of peripheral neuropathies. J Neuroimmunol 190:8-17.

Di Paola M, Di Iulio F, Cherubini A, Blundo C, Casini AR, Sancesario G, Passafiume D, Caltagirone C, Spalletta G (2010) When, where, and how the corpus callosum changes in MCI and AD: a multimodal MRI study. Neurology 74:1136-1142.

Harold D, Abraham R, Hollingworth P, Sims R, Gerrish A, Hamshere ML, Pahwa JS, Moskvina V, Dowzell K, Williams A, Jones N, Thomas C, Stretton A, Morgan AR, Lovestone S, Powell J, Proitsi P, Lupton MK, Brayne C, Rubinsztein DC, et al (2009) Genome-wide association study identifies variants at CLU and PICALM associated with Alzheimer's disease. Nat Genet 41:1088-1093.

Ho AJ, Stein JL, Hua X, Lee S, Hibar DP, Leow AD, Dinov ID, Toga AW, Saykin AJ, Shen L, Foroud T, Pankratz N, Huentelman MJ, Craig DW, Gerber JD, Allen AN, Corneveaux JJ, Stephan DA, DeCarli CS, DeChairo BM, et al (2010a) A commonly carried allele of the obesity-related FTO gene is associated with reduced brain volume in the healthy elderly. Proc Natl Acad Sci U S A 107:8404-8409.

Ho AJ, Raji CA, Becker JT, Lopez OL, Kuller LH, Hua X, Dinov ID, Stein JL, Rosano C, Toga AW, Thompson PM (2010b) The effects of physical activity, education, and body mass index on the aging brain. Hum Brain Mapp. Advance online publication. Retrieved April 7, 2011. doi:101002/hbm21113.

Ho AJ, Raji CA, Becker JT, Lopez OL, Kuller LH, Hua X, Lee S, Hibar D, Dinov ID, Stein JL, Jack CR Jr, Weiner MW, Toga AW, Thompson PM, the ADNI Consortium, the CHS Consortium (2010c) Obesity is linked with lower brain volume in $700 \mathrm{AD}$ and MCI patients. Neurobiol Aging 31:1326-1339.

Hoeft F, Barnea-Goraly N, Haas BW, Golarai G, Ng D, Mills D, Korenberg J, Bellugi U, Galaburda A, Reiss AL (2007) More is not always better: increased fractional anisotropy of superior longitudinal fasciculus associated with poor visuospatial abilities in Williams syndrome. J Neurosci 27:11960-11965.

Honea RA, Vidoni E, Harsha A, Burns JM (2009) Impact of APOE on the healthy aging brain: a voxel-based MRI and DTI study. J Alzheimers Dis 18:553-564

Hua X, Leow AD, Parikshak N, Lee S, Chiang MC, Toga AW, Jack CR Jr, Weiner MW, Thompson PM (2008) Tensor-based morphometry as a neuroimaging biomarker for Alzheimer's disease: an MRI study of 676 AD, MCI, and normal subjects. Neuroimage 43:458-469.

Jackson DN (1984) MAB, multidimensional aptitude battery: manual. Port Hurton, MI: Research Psychologists.

Jahanshad N, Lee AD, Barysheva M, McMahon KL, de Zubicaray GI, Martin NG, Wright MJ, Toga AW, Thompson PM (2010) Genetic influences on brain asymmetry: a DTI study of 374 twins and siblings. Neuroimage 52:455-469.

Kang HM, Zaitlen NA, Wade CM, Kirby A, Heckerman D, Daly MJ, Eskin E (2008) Efficient control of population structure in model organism association mapping. Genetics 178:1709-1723.

Kohannim O, Hua X, Hibar DP, Lee S, Chou YY, Toga AW, Jack CR Jr, Weiner MW, Thompson PM (2010) Boosting power for clinical trials using classifiers based on multiple biomarkers. Neurobiol Aging 31:1429-1442.

Lambert JC, Heath S, Even G, Campion D, Sleegers K, Hiltunen M, Combarros O, Zelenika D, Bullido MJ, Tavernier B, Letenneur L, Bettens K, Berr C, Pasquier F, Fiévet N, Barberger-Gateau P, Engelborghs S, De Deyn P, Mateo I, Franck A, et al (2009) Genome-wide association study identifies variants at CLU and CR1 associated with Alzheimer's disease. Nat Genet 41:1094-1099. 
Lee JE, Chung MK, Lazar M, DuBray MB, Kim J, Bigler ED, Lainhart JE, Alexander AL (2009) A study of diffusion tensor imaging by tissuespecific, smoothing-compensated voxel-based analysis. Neuroimage 44:870-883.

Leow A, Huang SC, Geng A, Becker J, Davis S, Toga A, Thompson P (2005) Inverse consistent mapping in 3D deformable image registration: its construction and statistical properties. Inf Process Med Imaging 19:493-503.

Lepore N, Chou YY, Lopez OL, Aizenstein HJ, Becker JT, Toga AW, Thompson PM (2008) Fast 3D fluid registration of brain magnetic resonance images. In: Medical Imaging 2008: Physiology, Function, and Structure from Medical Images. San Diego, USA: SPIE.

Liu Y, Spulber G, Lehtimaki KK, Kononen M, Hallikainen I, Grohn H, Kivipelto M, Hallikainen M, Vanninen R, Soininen H (2009) Diffusion tensor imaging and tract-based spatial statistics in Alzheimer's disease and mild cognitive impairment. Neurobiol Aging. Advance online publication. Retrieved April 11, 2011. doi:10.1016/j.neurobiolaging.2009.10.006.

Meyer-Lindenberg A, Nicodemus KK, Egan MF, Callicott JH, Mattay V, Weinberger DR (2008) False positives in imaging genetics. Neuroimage 40:655-661.

Nierenberg J, Pomara N, Hoptman MJ, Sidtis JJ, Ardekani BA, Lim KO (2005) Abnormal white matter integrity in healthy apolipoprotein E epsilon4 carriers. Neuroreport 16:1369-1372.

Nucifora PG, Verma R, Lee SK, Melhem ER (2007) Diffusion-tensor MR imaging and tractography: exploring brain microstructure and connectivity. Radiology 245:367-384.

Persson J, Lind J, Larsson A, Ingvar M, Cruts M, Van Broeckhoven C, Adolfsson R, Nilsson LG, Nyberg L (2006) Altered brain white matter integrity in healthy carriers of the APOE epsilon4 allele: a risk for AD? Neurology 66:1029-1033.

Raji CA, Ho AJ, Parikshak NN, Becker JT, Lopez OL, Kuller LH, Hua X, Leow AD, Toga AW, Thompson PM (2010) Brain structure and obesity. Hum Brain Mapp 31:353-364.

Resnick SM, Sojkova J, Zhou Y, An Y, Ye W, Holt DP, Dannals RF, Mathis CA, Klunk WE, Ferrucci L, Kraut MA, Wong DF (2010) Longitudinal cognitive decline is associated with fibrillar amyloid-beta measured by [11C]PiB. Neurology 74:807-815.

Ringman JM, O'Neill J, Geschwind D, Medina L, Apostolova LG, Rodriguez Y, Schaffer B, Varpetian A, Tseng B, Ortiz F, Fitten J, Cummings JL,
Bartzokis G (2007) Diffusion tensor imaging in preclinical and presymptomatic carriers of familial Alzheimer's disease mutations. Brain 130:1767-1776.

Roth AD, Ramírez G, Alarcón R, Von Bernhardi R (2005) Oligodendrocytes damage in Alzheimer's disease: beta amyloid toxicity and inflammation. Biol Res 38:381-387.

Rovio S, Kåreholt I, Helkala EL, Viitanen M, Winblad B, Tuomilehto J, Soininen H, Nissinen A, Kivipelto M (2005) Leisure-time physical activity at midlife and the risk of dementia and Alzheimer's disease. Lancet Neurol 4:705-711.

Salat DH, Tuch DS, van der Kouwe AJ, Greve DN, Pappu V, Lee SY, Hevelone ND, Zaleta AK, Growdon JH, Corkin S, Fischl B, Rosas HD (2010) White matter pathology isolates the hippocampal formation in Alzheimer's disease. Neurobiol Aging 31:244-256.

Scarmeas N, Luchsinger JA, Schupf N, Brickman AM, Cosentino S, Tang MX, Stern Y (2009) Physical activity, diet, and risk of Alzheimer disease. JAMA 302:627-637.

Smith CD, Chebrolu H, Andersen AH, Powell DA, Lovell MA, Xiong S, Gold BT (2010) White matter diffusion alterations in normal women at risk of Alzheimer's disease. Neurobiol Aging 31:1122-1131.

Stricker NH, Schweinsburg BC, Delano-Wood L, Wierenga CE, Bangen KJ, Haaland KY, Frank LR, Salmon DP, Bondi MW (2009) Decreased white matter integrity in late-myelinating fiber pathways in Alzheimer's disease supports retrogenesis. Neuroimage 45:10-16.

Thomason ME, Thompson PM (2011) Diffusion imaging, white matter and psychopathology. Annu Rev Clin Psychol 7:63-85.

Wechsler D (1981) Wechsler adult intelligence scale-revised. San Antonio, TX: The Psychological Corporation.

Zhang Y, Schuff N, Du AT, Rosen HJ, Kramer JH, Gorno-Tempini ML, Miller BL, Weiner MW (2009) White matter damage in frontotemporal dementia and Alzheimer's disease measured by diffusion MRI. Brain 132:2579-2592.

Zlokovic BV, Martel CL, Matsubara E, McComb JG, Zheng G, McCluskey RT, Frangione B, Ghiso J (1996) Glycoprotein 330/megalin: probable role in receptor-mediated transport of apolipoprotein $\mathrm{J}$ alone and in a complex with Alzheimer disease amyloid beta at the blood-brain and blood-cerebrospinal fluid barriers. Proc Natl Acad Sci U S A 93:4229-4234. 